\title{
ArcheoSciences
}

Revue d'archéométrie

\section{Richard, H, Magny, M. et Mordant, Cl. (dir.), Environnements et cultures à l'Âge du Bronze en Europe occidentale}

\section{Christine Chaussé}

\section{(2) OpenEdition \\ 1 Journals}

Édition électronique

URL : https://journals.openedition.org/archeosciences/1052

DOI : 10.4000/archeosciences. 1052

ISBN : 978-2-7535-1597-0

ISSN : $2104-3728$

\section{Éditeur}

Presses universitaires de Rennes

\section{Édition imprimée}

Date de publication : 31 décembre 2008

Pagination : 153-154

ISBN : 978-2-7535-0868-2

ISSN : $1960-1360$

\section{Référence électronique}

Christine Chaussé, «Richard, H, Magny, M. et Mordant, Cl. (dir.),

Environnements et cultures à l'Âge du Bronze en Europe occidentale », ArcheoSciences [En ligne], 32 | 2008, mis en ligne le 31 décembre 2008, consulté le 21 septembre 2021. URL : http://

journals.openedition.org/archeosciences/1052; DOI : https://doi.org/10.4000/archeosciences.1052 


\title{
Compte rendu de lecture
}

\author{
Richard, H, Magny, M. et Mordant, Cl. (dir.), 2007. Environnements et cultures à l'Âge du Bronze en Europe occiden- \\ tale, Paris, CTHS, 400 p.
}

Cet ouvrage rassemble un ensemble de vingt-quatre contributions présentées dans le cadre du $129^{\circ}$ Congrès national des sociétés historiques et scientifique, congrès organisé par le Comité des travaux historiques et scientifiques. Ce colloque qui s'est tenu à Besançon en 2004, clôturait un programme de recherche ECLIPSE, développé entre 2002 et 2004 et consacré aux "emprises et déprises agricoles, expansion et régression des sociétés entre 3500 et $2500 \mathrm{BP}$ en Europe occidentale : déterminisme climatique ou/et phénomènes socio-culturels ». Ce programme est né de la volonté de combler le déficit des connaissances sur les interactions Hommes-Milieux au cours de l'Âge du Bronze et de rattraper le niveau des acquis obtenus pour les sociétés du Néolithique et de l'Âge du Fer sur les mêmes problématiques. La thématique est présentée à l'échelle de l'Europe occidentale, ainsi que le souligne le titre du n ${ }^{\circ} 21$ des Documents Préhistoriques intitulé «Environnements et cultures à l'Âge du Bronze en Europe occidentale ». Effectivement huit articles sont consacrés à des études qui ont été menées en Allemagne (Alpes), Espagne (Pyrénées), Suisse (Alpes), Pays-Bas (delta Rhin-Meuse), Italie (Plaine du Pô, côte adriatique) et Albanie (Balkans). Les seize autres sont des études réparties en France avec une distribution préférentielle marquée autour d'un pôle Alpes-Jura-Rhône qui rassemble sept articles, contre trois pour le littoral Atlantique (Normandie, Bretagne, Médoc), deux pour le bassin de Paris (Lorraine, Île de France-Bourgogne), deux également pour le Massif Central, et un pour les Pyrénées. Les contributions sont principalement écrites en français, mais comptent également trois articles en langue étrangère dont l'allemand, l'anglais et l'italien. Toutes proposent en fin de texte un résumé bilingue (français/anglais) voire trilingue (allemand). Les illustrations, principalement en noir et blanc, sont globalement de bonne qualité et correctement légendées. On peut cependant regretter que certains articles soient dépourvus de cartes de localisation générale, même si la lacune est compensée dans l'introduction de l'ouvrage par une localisation des sites évoqués à l'échelle de l'Europe de l'ouest. Aussi, il aurait été avantageux, dans un esprit d'homogénéisation du discours portant sur la chronologie des événements et leur succession d'une région à l'autre, d'uniformiser la présentation des datations ${ }^{14} \mathrm{C}$, données selon les articles en BP, BC, BC cal. ou BP cal.

Comme expliqué dans une introduction claire et bien détaillée, signée par $\mathrm{H}$. Richard, $\mathrm{M}$. Magny et $\mathrm{Cl}$. Mordant, ces communications puis leur publication dans le présent ouvrage proposent de reconsidérer les savoirs sur les sociétés de l'Âge du Bronze en Europe occidentale à la lumière des apports les plus récents sur l'étude des paléoenvironnements. En effet, les connaissances sur l'Âge du Bronze européen, jusque-là réunies, font état d'une tripartition chronoculturelle (Bronze ancien, moyen, final) dans laquelle le Bronze moyen (XvII ${ }^{\mathrm{e}}$-XIV ${ }^{\mathrm{e}}$ siècle av. J.-C.) apparaît comme une période de déprise agricole, voire un repli des sociétés. Elle coïncide avec une détérioration climatique marquée par une augmentation globale de l'humidité. Le croisement des données sociétales et paléoenvironnementales permet-il de vérifier ces premiers constats établis indépendamment? Le cas échéant, doit-il être nuancé, des mécanismes de causalité peuvent-ils être dégagés? Ce travail de confrontation des données est présenté selon deux ensembles d'articles. Le premier rassemble sept contributions plutôt consacrées à la restitution du cadre paléoclimatique général (p. 13-120), le second, qui compte dix-sept articles, est dédié à la problématique emprise/déprise des occupations humaines de l'Âge du Bronze (p. 121-395), abordées à travers le prisme d'analyses paléoenvironnementales ou socio-culturelles.

Les sept premiers articles attachés à la restitution du cadre paléoclimatique, sont surtout centrés autour du pôle AlpesJura-Rhone (Magny et al., Holzhauser, Chapron et al., Billamboz, Richard et Gauthier, Court-Picon et al.) avec une incursion pyrénéenne (Galop et al.). Le cadre est établi à partir d'études litho-chronostratigraphiques sur les remplissages lacustres (Magny et al., Chapron et al.) mais aussi fluviatiles (Rhône : Berger et al., delta Rhin-Meuse : Jongste et Van Zijverden, contributions apparaissant dans le volet "problématiques archéologiques thématiques »), glaciologiques sur les dépôts morainiques (Holzhauser), dendrochronologique/dendro-écologiques sur vestiges ligneux anthropiques ou non (Billamboz; Holzhauser), palynostratigraphiques 
(Richard et Gauthier, Court-Picon et al., Galop et al.). Les travaux présentés proposent un affinement du cadre chronoclimatique sur la base de la confrontation et du croisement de différents indicateurs. Cette confrontation permet de valider la tripartition climatique de la période avec une dégradation des conditions globales placée entre 1800/1700 - 1350 cal BC qui enveloppe le Bronze moyen. Les travaux futurs devront, de l'avis des auteurs, poursuivre l'acquisition les données chronologiques afin de tendre vers une meilleure résolution des phases de transition climatique.

Les articles thématiques qui suivent, au nombre de dixsept, s'attachent à la reconnaissance de la signature du phénomène emprise/déprise humaine à partir d'une lecture analytique interdisciplinaire de certains faits culturels ou indicateurs environnementaux à signification anthropique (pollen, graine, charbon, aménagement/perturbation hydrologique, crise érosive...). Ainsi, certaines données polliniques (Richard et Gauthier, Galop et al.), de géomorphologie fluviale (Berger et al., Jongste et van Zijverden) ou certains éléments de la culture matérielle (Georges et Cubizolles) s'accordent à reconnaître une déprise humaine au cours du Bronze moyen en relation avec une humidité plus abondante. Toutefois, l'analyse est nuancée chez certains auteurs (Galop et al.), voire révisée chez d'autres (Court-Picon et al.) qui observent une continuité des occupations humaines et privilégient plus une réorganisation socio-culturelle des espaces agro-pastoraux qu'un repli des sociétés. La question est également abordée, d'un point de vue démographique d'après la densité des sites, ou l'étude de bilans sédimentaires (Billaud et al., Pétrequin et Weller, Milcent et MennessierJouannet). Si pour Billaud et al., les rives des lacs alpins sont effectivement désertées au cours du $\mathrm{II}^{e}$ millénaire, les autres auteurs, dont les travaux ne sont pas strictement limités au domaine lacustre, analysent leurs données en termes de poussée démographique et de réorganisation spatiale des sociétés, ce en continuité d'une évolution amorcée au Bronze ancien et qui se poursuit jusqu'au Bronze final. On retrouve la même idée chez Marcigny et al., Riera et al., Gabillot et al., Di Fraia et Koenig et Ruffaldi, qui enregistrent une occupation continue des espaces naturels à partir d'analyses sédimentaires, polliniques croisées avec d'autres indicateurs notamment biologiques (faune, charbons, graines), mais également culturels comme la métallurgie, ce qui est novateur. Certains auteurs (Roussot-Laroque, Cremaschi) montrent même que localement, le Bronze moyen se traduit par une période de mise en valeur agricole de terres désormais bien drainées. Une part de ces mutations culturelles qui s'expriment dans la production matérielle (Hafner et Sutter) et artistique (de Saulieu) est-elle alors induite par des apports migratoires comme le suggère Mordant et al. ?

Le foisonnement des approches et la diversité des analyses tant géographiques que thématiques font de cet ouvrage un incontournable pour cette période de la préhistoire récente de l'Europe occidentale. On peut regretter cependant que le bassin de la Seine, riche en données notamment, n'ait pas été inclus dans cette réflexion. Quoi qu'il en soit, le résumé analytique que nous faisons de l'ouvrage est loin de rendre compte de tous les aspects développés mais aussi de certaines positions avancées par les auteurs. Si de prime abord, l'essentiel des articles revisite l'Âge du Bronze sous l'angle de la continuité et non plus de la rupture, apparait en filigrane une réflexion sur l'adaptabilité des populations au changement de leur milieu, à la réponse qu'elles ont fournie, aux conséquences possibles de ces réponses sur leur milieu naturel, sur l'organisation de leur société et celle de leurs voisines.

Christine Chaussé

INRAP Centre Île-de-France UMR 8591 Laboratoire de Géographie Physique 1 place Aristide Briand 92195 Meudon cedex Christine.Chausse@cnrs-bellevue.fr 